\title{
Dynamic Focus Capture Technology and Its Application in Pedestrian Flow Statistics System
}

\author{
Shoujin Wang ${ }^{1, a^{*}}$, Tianbo Liu ${ }^{1, b}$ and Shengbin Zhao ${ }^{1, c}$ \\ ${ }^{1}$ School of Information \& Control engineering, Shenyang Jianzhu University, Shenyang, China \\ a23917240@qq.com, b49357122@qq.com, ${ }^{c} 29171840 @ q q . c o m$
}

*The corresponding author

Keywords: Dynamic focus capture; Pedestrian flow; Motion capture technology; Statistics system

\begin{abstract}
Dynamic focus capture technology is an important research field in computer vision technology and has broad application prospects. From data acquisition and motion capture, this paper proposes a method of obtaining a dynamic focus by moving target single camera for motion recognition. After a series of demonstration tests, the dynamic focus capture technology that has excellent applicability and broad prospects in pedestrian flow statistics system has been verified
\end{abstract}

\section{Introduction}

This paper studies how to achieve the target moving object or motion detection and capture based on single camera-based hardware. System input device used in an ordinary camera that can capture images and color information. Using color information through the centroid algorithm, to capture the position of the target object and obtain sports focus [1]. Separate analysis location and range of variation over time can determine the focus of the action to be captured has happened or not.

In the detection of moving targets, first to separate the detected objects from the background with background subtraction and then filter a region and a color, the final target to be detected will have a high degree of recognition [2]. Therefore, this system requires a big difference on the object to be tested with the background color to improve recognition accuracy. Without affecting the accuracy of the result of the capture of the premise, it should try to reduce the image size can be calculated to ensure that the focus of the process and less time consuming. Finally, the system is implemented with Visual Studio 2010.

The innovations of this paper are:

(1) Less require on hardware requirements. The current cost of the camera is being reduced year by year, the majority of mobile phones and laptops comes with enough pixel camera can be used as an image input source of the system. In addition, it does not require additional special hardware, such as an infrared transceiver, motion capture glove high-performance computers and so on.

(2) Suitable for different conditions. It is relatively simple to focus data as the basis for further processing and does not require knowledge of mathematical modeling and the learning process, as a basis for a secondary application development.

\section{The Aim and Significance of the Search}

IVS, Intelligent Video Surveillance, is a new emerging field of computer vision, which involves image processing, image analysis many areas of research, machine vision, pattern recognition, artificial intelligence, is a comprehensive interdisciplinary problem and a challenging topics at the forefront. Pedestrian traffic statistics system is an intelligent video surveillance system as an important measurement tool in the Commercial Applications [3, 4]. Compared with the traditional manual counting mode, human traffic statistics intelligent video surveillance system of the integration of information technology will be able to provide timely and accurate figures and help to carry out scientific analysis and efficient management, allowing IT technology to plug wings for the economic development. "Pedestrian flow statistics system" described in this chapter is based on 
dynamic focus technology capture a Pedestrian flow statistics software system, with the advantages of simple configuration, adaptability and so on[5,6].

\section{Design Ideas}

Pedestrian flow statistics system is the software systems that use camera surveillance personnel movements and static the flow of Pedestrian within the scope of monitor. Among them, the situation of camera captures monitoring personnel movements is through the introduction of dynamic focus capture technology package library implemented, the range of person entry into camera will be monitored to focus a large dynamic quality [7, 8]. By analyzing the quality and trajectory of this dynamic focus, combined with relevant judgment algorithm, the Pedestrian flow statistics can be achieved.

Since the operation mechanism of dynamic focus technology limitations, the tracking ability is not strong for small targets of the system. Therefore, it is suitable for horizontal viewing angle and close monitoring angle, the design of the system will also optimize the viewing angle.

\section{Function Partition}

According to the software demands, the system functions can be partitioned as follows:

(1) Launch. Initializing related code and launch the camera.

(2) Exit. Exit the software and release all system resources.

(3) Set the background. Set the background image of the Dynamic Focus Capture System.

(4) Adjustment of threshold value / sensitivity. Adjust the operation conditions to fine-tune recognition.

(5) Monitoring start (end). The captured process of start (end) motion.

(6) Monitoring pause (resume). The captured process of pause (resume) motion.

(7) About. Introduce software developers' information and version information.

\section{Core Functions Design}

Function Introduction. The core function of this system is to calculate the Pedestrian flow in horizontal, which the crowd on the two horizontal directions will be calculated respectively. For a person through the camera and is determined to be an effective personnel through the process shown in Fig. 1. Monitoring process will be achieved through separate thread loop running for some code to implement monitoring, pedestrian flow statistics results will be displayed on the system interface.

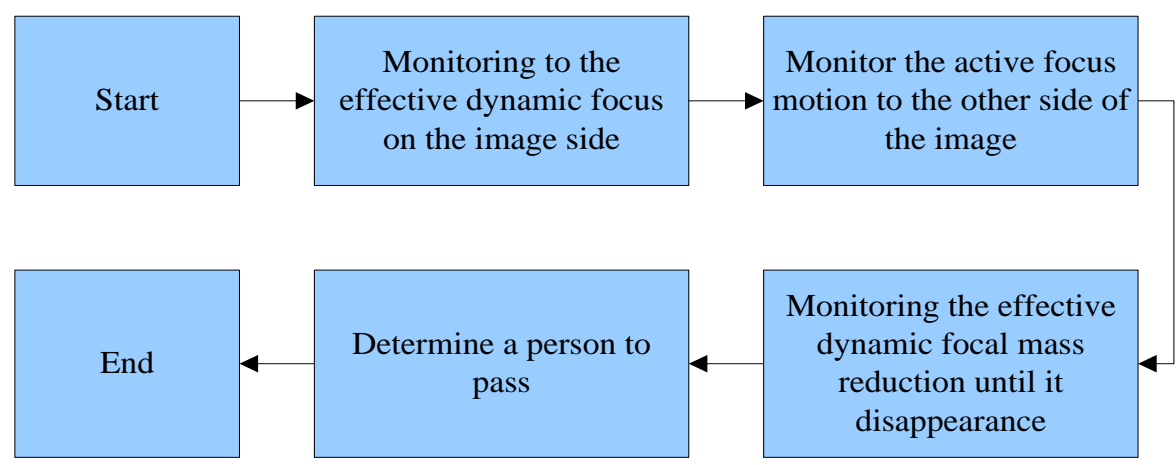

Figure 1. Personnel passing decision process schematic

Module Allocation. The main module allocations are as follows:

(1) Camera control module. Using Windows API to call computer's camera, with functions of the camera turned on, turn off the camera, shots into the buffer and so on. 
(2) Image processing module. Provide incoming gray scale image array operation, providing focus comparison operations as well as provide obtaining external focus information methods.

(3) Thread control module. It provides multithreaded template that can be implemented by secondary developers. Provide multithreaded start, suspend, resume, termination and other operations.

(4) Adjustment module. Provide methods of adjusting relevant system parameters.

(5) Adapter module. It provides quick access to other features of the interface module and cooperates with other modules.

Interface Design. (1) User interface. In the user interface portion, according to the results of the needs analysis, users need a user-friendly interface. The interface design should be simple and clear, easy to operate, and the layout of the interface needs to be considered. It should highlight the important display an error message. At the same time, the error message should be given in standard form when meets the error. Overall, the system user interface should have reliability, simplicity and is easy to learn and use.

(2) External interface. Use Windows API to access and control the camera.

(3) Internal interface. Each module provides functions for adaptation module interfaces. The secondary adapter module provides high-level interfaces for developers.

Run-time Control. (1) Operation Control will strictly follow the calling relationships among modules function to achieve. In each transaction module, the need for proper control operation judgment to select the correct operation of the control path.

(2) Runtime system efficiency greater requirements, image processing speed is slow cause loss of information, computing speed dynamic focus directly affect the use effect is applied.

Processing (3) Beware of multithreading resources between threads call the conflict and logic errors.

Detailed Design. Input: Justify threshold and thread control command.

Algorithm description: Pseudo code of the core functions is described as follows:

Cyclic execution of the code segment

Enter the current frame for the frame to be calculated

Computational dynamic focus

If (find a target)

If (the focus becomes smaller)

Loss index plus 1

If (loss index > 3)

\{

Marked: not found

Corresponding direction of the flow of people +1

\}

\}

Or

\{

Focus information

Loss index gives 0

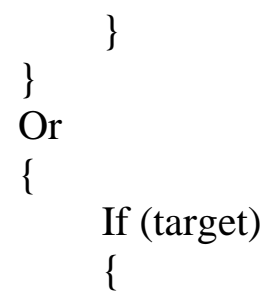

Mark: find the target 


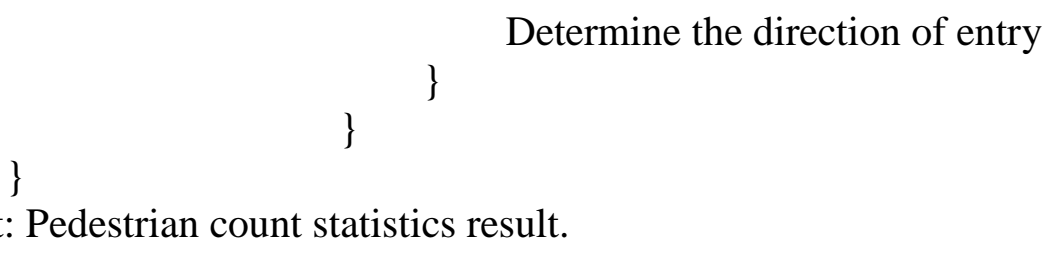

Output: Pedestrian count statistics result.

\section{Operation Result}

Open the main interface and switch on the camera. The results are shown in Fig. 2.

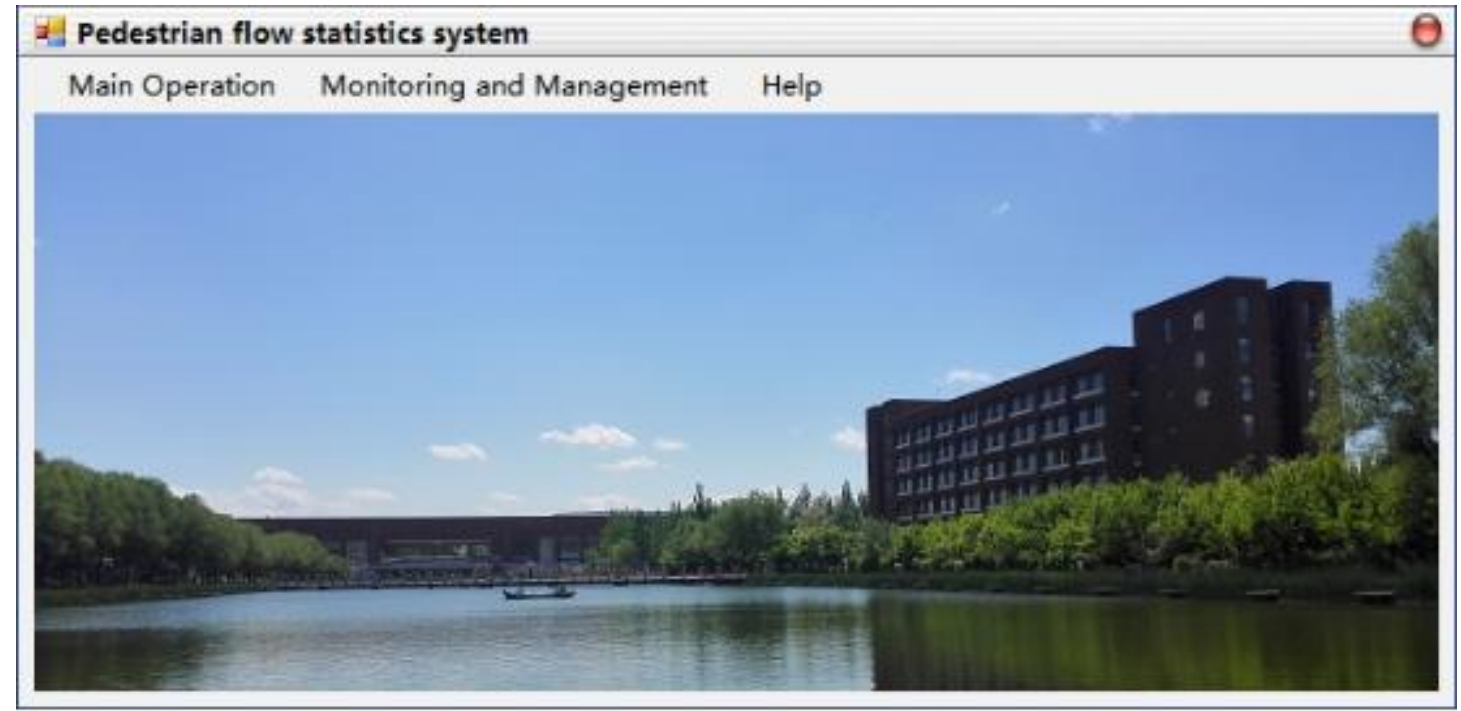

Figure 2. Main interface and camera real-time window

Set the background. When detecting, the main interface displays screen count and identifies the traveling direction arrow. The specific results are shown in Fig. 3.

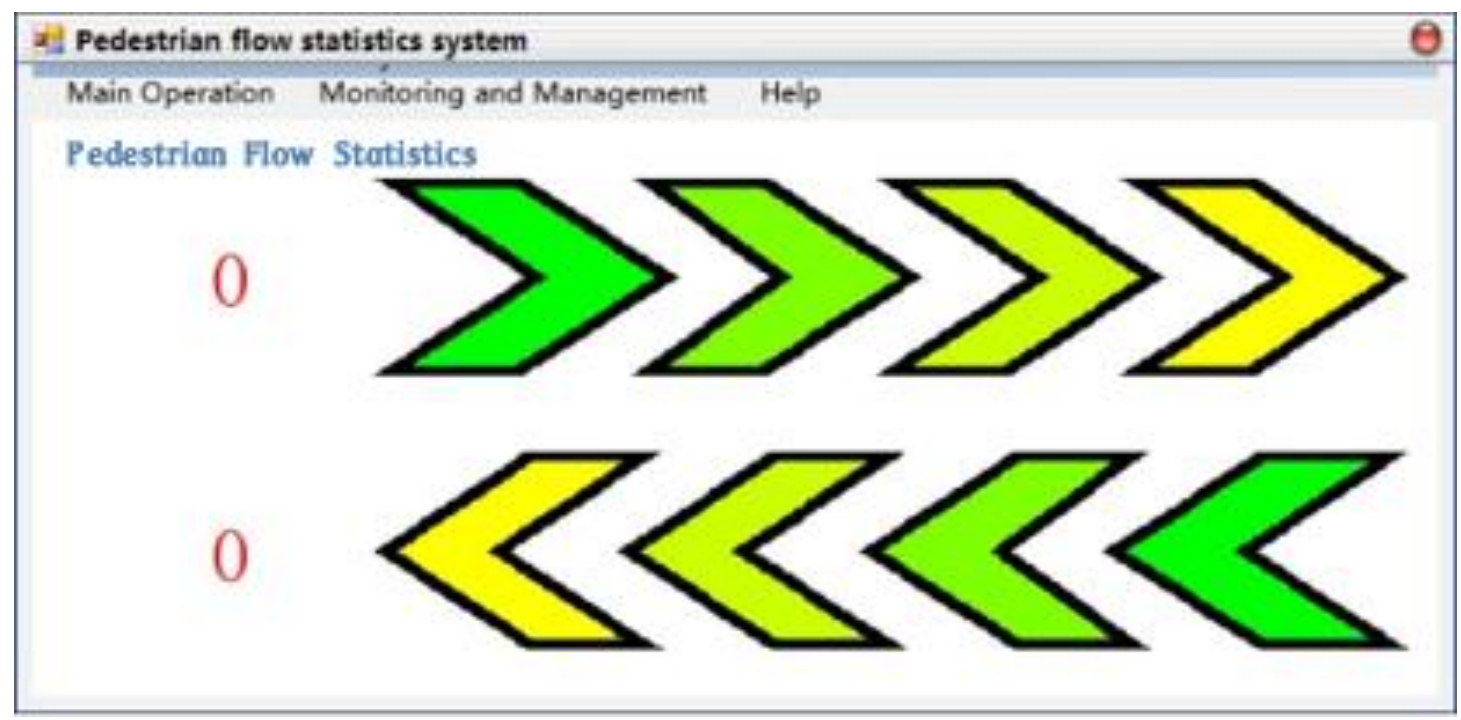

Figure 3. Shortcut of monitoring progress (No Pedestrian passing)

In the test, 7 Pedestrian were arranged in two directions sequentially through individual camera from the camera about $30 \mathrm{~cm}$. System statistics is shown in Fig. 4.

Test results: Through testing, this system could work properly. It could determine sensitively, accuracy in Pedestrian counting, achieve the desired objectives. During the test, the statistics could be inaccurate sometimes when many pedestrian entering in a short time or sometimes due to the algorithm restrictions, the system needs to be improved further. 


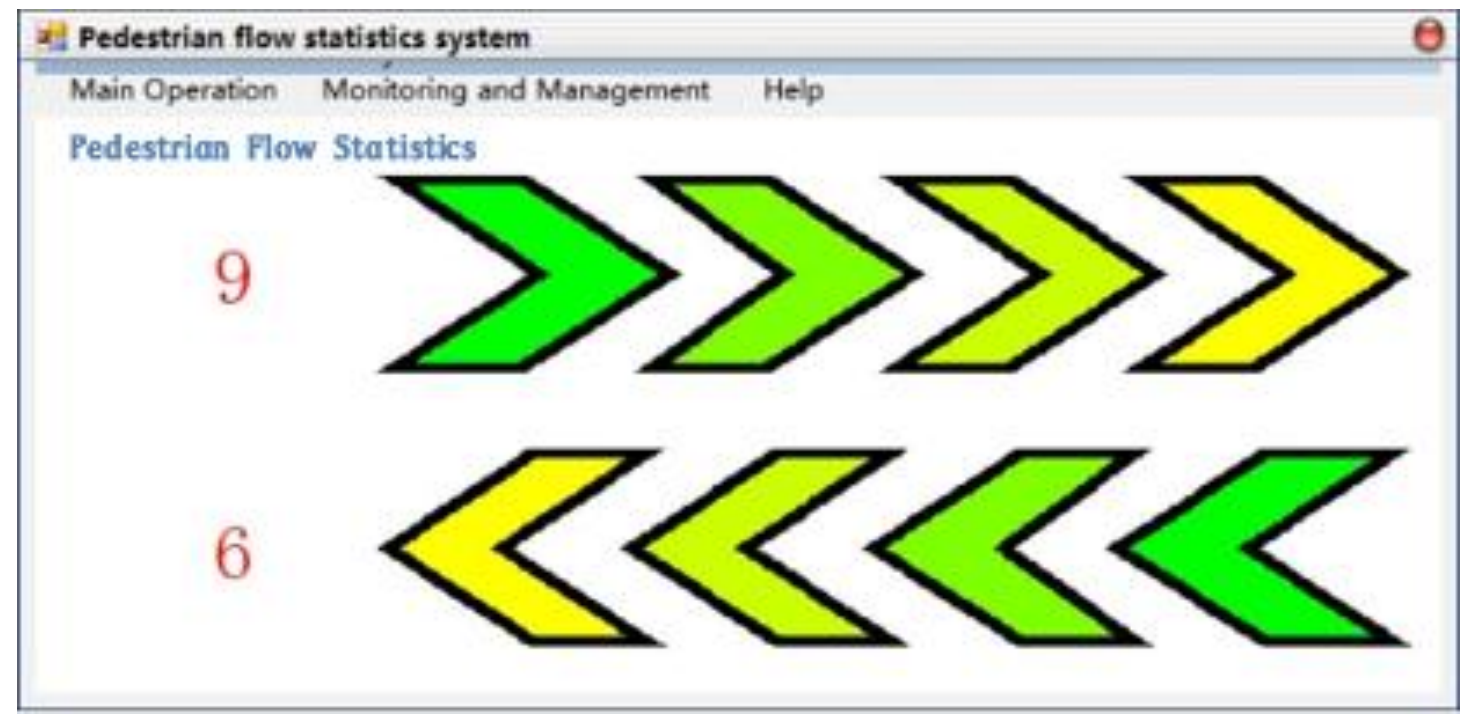

Figure 4. Shortcut of monitoring progress (After Pedestrian passed)

\section{Conclusions}

Although the focus of dynamic capture technology has made some progress, but it is still in its infancy, there are many issues have not been resolved and there is much room for improvement. The current advantages of this technology are low hardware cost, simple structure, low cost secondary development study and performs well in a particular mission. The disadvantage of this technology lies that there is no effective action for complex motion and the recognition of multiple moving targets and overlapping targets is not high, as well as higher external environment.

\section{References}

[1] Shoujin Wang, Yang Cao, Jingang Shi. Dynamic Focus Capture Method and Its Application[C]// 6th International Conference on Electronic, Mechanical, Information and Management Society. 2016.

[2] Min Lin, Bin Li, Qiao-Hong Liu.Identification of Eye Movements from Non-front--al Face Images for Eye-controlled Systems [J] International Journal of Automation \& C-omputing,2014,issue No.5: 29 30

[3] Shoujin Wang, Ziyang Han, Song Guo. Design and Implementation on Gesture Recognition Game Controller[C]// 6th International Conference on Electronic, Mechanical, Information and Management Society. 2016.

[4] Xinchen Liu, Huiyuan $\mathrm{Fu}$ and Huadong Ma. Real-time tracking and gesture recognition gesture-based RGB-D camera [D] Beijing: Beijing Youdian University, 2014: 13 14

[5] Yaping Gao. Study based on single camera gesture recognition method [D].Xiamen: Xiamen University, 2014: 34 35

[6] Van Rhijn, Gu Johanna Willemina, Bosch, Tim, Könemann, Reinier. Method and system for posture evaluation: EP2508127 [P]. 2012.

[7] Fei Liu. Human action recognition based on Kinect skeleton information [D].Shanghai: Shanghai Donghua University, 2014: 25 27

[8] Leu J S, Tung N H. Design and Implementation of a Reconfigurable Mobile Game Controller on Smartphone [J]. Wireless Personal Communications, 2014, 74(2):823-833. 